\title{
Idiopathic Guttate Hypomelanosis: A Comprehensive Overview
}

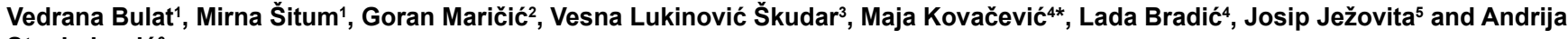
Stanimirović6

${ }^{1}$ Department of Dermatology and Venereology, University Hospital Center “Sestre milosrdnice", Vinogradska cesta 29, Zagreb, Croatia

${ }^{2}$ Naftalan Special Hospital for Medical Rehabilitation, Omladinska 23a, Ivanić Grad, Croatia

${ }^{3}$ Medical University Zagreb, Šalata 3, Zagreb, Croatia

${ }^{4}$ University Hospital Center "Zagreb“, Kišpatićeva 12, Zagreb, Croatia

${ }^{5}$ Centre for Croatian Studies, University of Zagreb, Borongajska cesta 83d, Zagreb, Croatia

${ }^{6}$ Department of Clinical Medicine, University of Applied Health Sciences, Mlinarska 38, Zagreb, Croatia

\begin{abstract}
Idiopathic guttate hypomelanosis (IGH) is a very common, acquired, and frequently ignored condition characterized by an appearance of multiple, well-circumscribed, asymptomatic, polygonal, white macules symmetrically distributed on the extensor forearms and shins. Once present, skin lesions do not increase in size with time and do not coalesce. IGH occurs in all races and skin types, especially in elderly patients over the age of 70 years. IGH appears to result from an impaired synthesis of melanin, decreased size and poor melanization of melanosomes and inadequate transfer of melanosomes from melanocytes to surrounding keratinocytes. The diagnosis of IGH is usually made clinically. Despite straightforward clinical appearance of IGH in majority of cases, several additional diagnostic procedures may be needed for confirmation of diagnosis in some less clear cases.
\end{abstract}

Keywords: Hypomelanosis; Melanocytes; Hypopigmentation;

\section{Introduction}

Hypomelanosis denotes an absence or reduction of melanin in the tissues, especially in the skin. Idiopathic Guttate Hypomelanosis (IGH), synonym hypomelanosis of Cummins and Cottel, is considered melanopenic hypomelanosis that may result from numerous and complex pathologic mechanisms. IGH does not appear to result from a reduction in the number of epidermal and/or follicular melanocytes, but rather from an impaired synthesis of melanin, decreased size and poor melanization of melanosomes and inadequate transfer of melanosomes from melanocytes to surrounding keratinocytes. The result of this process is rapid elimination of inactive melanocytes (Figure 1) [1,2].

IGH is a very common, acquired and frequently ignored condition. A positive correlation has been established between the incidence of this condition and aging. It is seen in up to $80 \%$ of individuals over the age of 70 years. The basic defect in IGH melanocytes may reflect the aging process [1]. The precise etiology of IGH is unknown, but a history of excessive, chronic sun exposure probably also plays a role for IGH is rarely seen in West Indians raised in England, but is common in those who spent their childhood in the Caribbean. According to Flabella et al. genetic influences have also been proposed as possible causes due to higher prevalence of IGH in the family of patients with IGH than in the control group [3].

IGH occurs in all races and skin types with a frequency ranging from 46 to $70 \%$. However, it is more striking in darker-skinned racial/ ethnic groups. In Caucasians, it may favour those with brown eyes and brown hair. An apparent female predominance is probably the result of heightened perception of a cosmetic problem.

\section{Clinical Features}

Multiple, well-circumscribed, asymptomatic, polygonal, white macules are symmetrically distributed on sun-exposed areas (Figures 2-5).

Skin lesions are most commonly seen on the extensor forearms and shins; the remainder of the extremities can be affected, but rarely the face (Figure 6). The lesions range usually from 2 to $8 \mathrm{~mm}$ in diameter and often delineated by the skin furrows, with occasional larger lesions up to $2.5 \mathrm{~cm}$. Once present, they do not increase in size with time and do not coalesce. The number of lesions increases with age. Hypomelanosis is an abnormality solely in the colour of the skin, with no texture abnormality. No spontaneous repigmentation has been observed. Vellus hairs within the lesions usually retain their pigment. Patients have usually signs of photoaging, including lentigines, and xerosis in the same involved areas [3].

\section{Diagnosis}

IGH is usually diagnosed clinically following a complete history and physical examination. Patients with IGH should be examined under both visible and ultraviolet light of about $365 \mathrm{~nm}$ wavelengths (i.e. Wood`s lamp).

While under visible light, the contrast between hypomelanosis and surrounding skin is not striking in light-skinned individuals, with Wood`s lamp examination more lesions may become apparent and a decrease in pigment is confirmed. Skin biopsy is not usually needed. Histologic examination of involved skin is usually most helpful for postinflammatory hypomelanosis such as sarcoidosis and mycosis fungoides, whereas in others (e.g. pityriasis lichenoides chronica) the histologic findings are often nonspecific [4].

\section{Histologic Features}

The most prominent histologic features of IGH are a flattening of the dermal-epidermal junction, moderate to marked reduction or focal absence of melanin granules in the basal and suprabasal layers, and a basket-weave hyperkeratosis $[5,6]$.

Immunohistochemical stains such as HMB45, Mel-5 or S100 reveal a moderate to relatively marked reduction of DOPA-positive epidermal

*Corresponding author: Maja Kovačević, University Hospital Center "Zagreb" Kišpatićeva 12, Zagreb, Croatia, Tel: 38513820077; E-mail: kovacevic.maja01@ gmail.com

Received: November 07, 2014; Accepted: November 12, 2014; Published: November 15, 2014

Citation: Bulat V, Šitum M, Maričić G, Škudar VL, Kovačević M, et al. (2014) Idiopathic Guttate Hypomelanosis: A Comprehensive Overview. Pigmentary Disorders 1:150. doi: 10.4172/2376-0427.1000150

Copyright: ( 2014 Bulat $\mathrm{V}$, et al. The terms of the Creative Commons Attribution License, which permits unrestricted use, distribution, and reproduction in any medium, provided the original author and source are credited. 


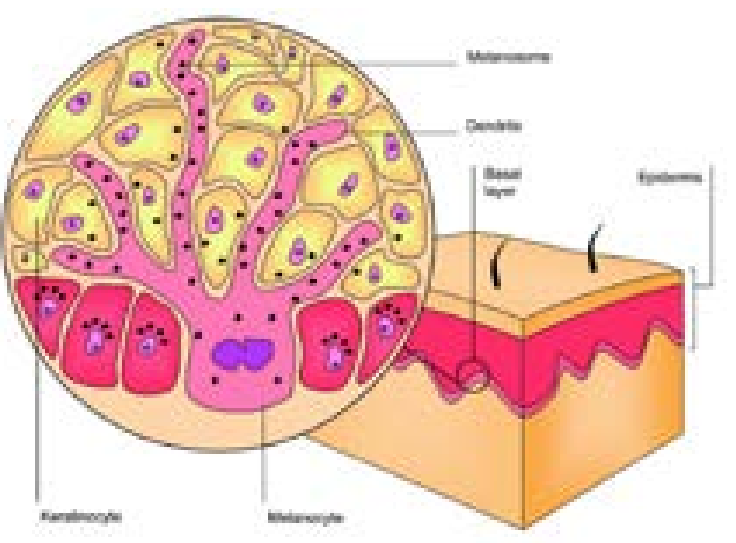

Figure 1: Structure of Melanocyte.

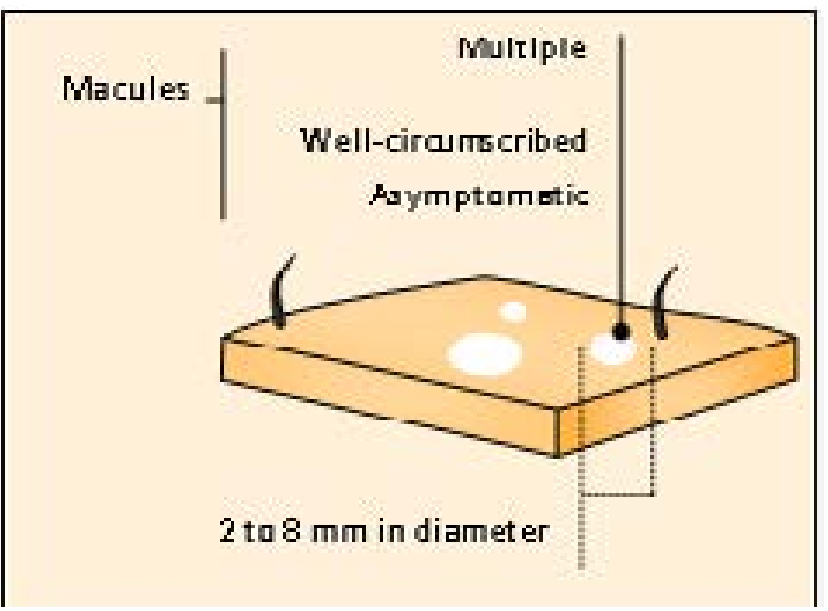

Figure 2: Multiple, White Macules, usually $2-8 \mathrm{~mm}$ in diameter on the Skin.

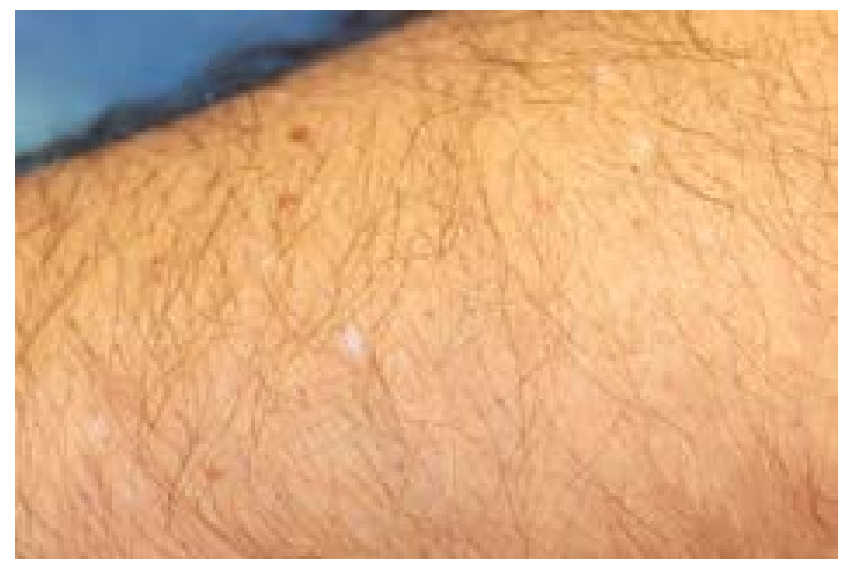

Figure 3: Multiple white macules photographed under visible light.

melanocytes (10-50\% compared with normal skin), which appear to be replaced by Langerhans' and dermal dendritic cells [2]. Collagen and elastic fibers are not affected. Electron microscopic studies are performed only for research purposes to characterize the subcellular events of melanogenesis because very few hypomelanotic disorders have a characteristic ultrastructural picture. By electron microscopy, the melanocytes in lesions are round and less dendritic with a decreased number of melanosomes in melanocytes with predominance of stages I (spherical melanosomes; no melanin deposition) and II (oval melanosomes; obvious matrix in the form of parallel longitudinal filaments; minimal deposition of melanin; high tyrosinase activity) and small sizes (Figure 7). The keratinocytes show a marked variation in melanin content, which may be absent or markedly decreased $[2,7,8]$.

\section{Differential Diagnosis}

The differential diagnosis of guttate leukoderma includes several clinical entities such as vitiligo, hypopigmented pityriasis versicolor or other cutaneous infections (e.g. leprosy, treponematoses, onchocerciasis, post kala-azar dermatosis), guttate morphea and guttate lichen sclerosus, postinflammatory hypopigmentation (e.g. pityriasis alba, secondary to psoriasis), tuberous sclerosis, achromic verrucae plana, pityriasis lichenoides chronica, sarcoidosis, hypopigmented mycosis fungoides, dyschromic amyloidosis and disseminated hypopigmented keratoses that follow photochemotherapy (Figure 8). Prior treatment with potent topical corticosteroids, azelaic acid and systemic medications (chloroquine, tyrosine kinase inhibitors) can also lead to hypomelanosis $[9,10]$.

A large number of infectious dermatoses can lead to guttate hypomelanosis. The clinical presentation of pityriasis versicolor differs enough from IGH to allow distinction. Pityriasis versicolor favors upper trunk, while IGH favors upper and lower extremities. The diagnosis of pityriasis versicolor is easily confirmed by potassium hydroxide $(\mathrm{KOH})$ examination of the associated scale, while macules in patients with IGH has smooth surface, without scales and negative $\mathrm{KOH}$ examination. A defect in melanosomal transfer to keratinocytes and decreased melanin synthesis are pathologic mechanisms seen in both IGH and pityriasis versicolor.

The clinical and histologic features of leprosy differ enough to allow distinction from IGH.

Although lesions of atrophie blanche favor the distal shins and ankles, these porcelain white scars are usually depressed and encircled by papular telangiectasias. The surface of lesions in IGH is smooth, but not atrophic.

Vitiligo can resemble macules in IGH. Macules in vitiligo are usually extending into patches, while macules in IGH are small in diameter and remain fixed. Spontaneous repigmentation has been observed in vitiligo, but not in IGH. Histologically, melanocytes are completely absent in vitiligo, while the number of melanocytes in IGH is usually normal [11].

Progressive macular hypomelanosis is distinguishable from IGH because macules are less white and less well-circumscribed on the trunk with rare progression on extremities. Electron microscopy of involved skin shows small melanosomes. This finding is also seen in IGH [12].

Chemically induced hypomelanosis from occupational exposure to depigmenting agents (such as phenol/catechol derivatives, sulfhydryls) is easily excluded by the history and physical examination from IGH. In the former lesions favor hands and forearms, whereas in the latter lesions favor extensor forearms and shins $[13,14]$.

IGH must be distinguished from the confetti-like lesions of tuberous sclerosis. The former condition is an acquired disorder of older adults, whereas the latter hypopigmented guttate macules are usually already present in childhood and adolescence. However, the neurologic findings observed in tuberous sclerosis are not associated with IGH [15]. 


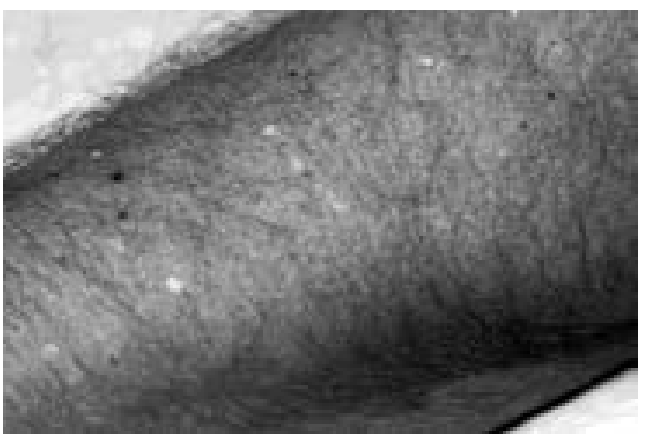

Figure 4: Multiple white macules photographed in black/white technique.

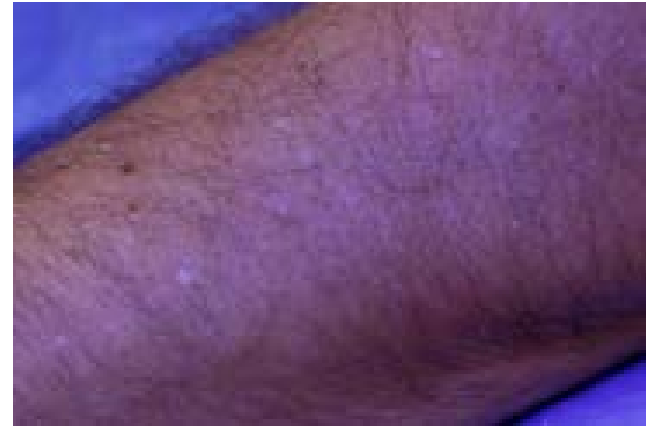

Figure 5: Multiple white macules photographed under WOOD light.

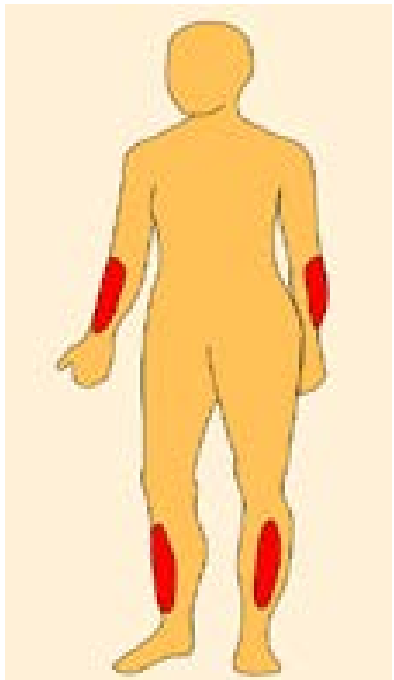

Figure 6: Multiple white macules photographed in black/white technique.

The most important entity in differential diagnosis for guttate, extragenital lichen sclerosus is morphea. Histologically, loss of elastic fibers is typical for lichen sclerosus and is not found in morphea.

Numerous inflammatory dermatoses can lead to guttate hypomelanosis. Cutaneous inflammation is able to interrupt melanosome transport from melanocytes to keratinocytes and alter melanin production, the number and size of melanosomes in the affected skin. The most common postinflammatory hypomelanosis is pityriasis alba that may resemble IGH. In the former the skin lesions are initially erythematous, slightly scaly, ill-defined macules, face is the most frequent site of involvement, the peak incidence occurs in childhood, whereas in the latter there is no erythema, no scales, macules are well-circumscribed, the face is rarely affected, and incidence of IGH increases with age [16]

Hypomelanotic lesions occur on the trunk, may be pruritic, and skin lesions are initially erythematous in patients with hypopigmented mycosis fungoides, while white macules in IGH are asymptomatic, symmetrically distributed on extremities, and there is no erythema. Routine histology shows the typical features of mycosis fungoides. Electron microscopy reveals numerous morphologically normal melanosomes in melanocytes, but there is a defect in melanosomal transfer to keratinocytes $[17,18]$ Clinically, sarcoidosis may resemble IGH. The lesions appear most commonly on the extremities, are asymptomatic, and have no other secondary changes. Spontaneous repigmentation has been observed in sarcoidosis, but not in IGH. Histologically, non-caseating granulomas are seen in the dermis of patients with sarcoidosis. The melanosomes appear morphologically normal in sarcoidosis, their number within keratinocytes is decreased, while melanosomes have several abnormalities in individuals with IGH [10].

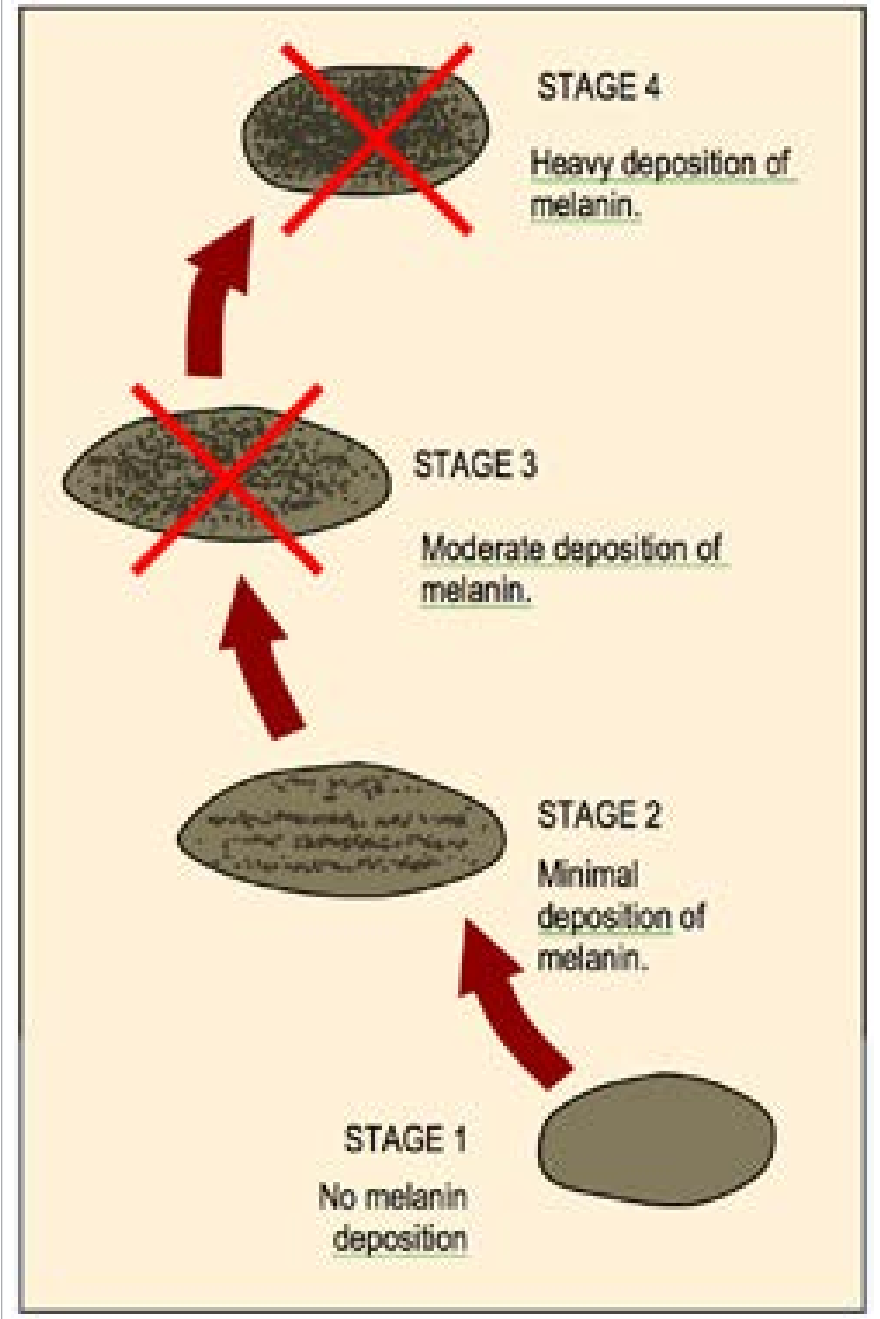

Figure 7: Predominance of stages I and II of eumelanin melanosomes in patients with IGH. 
Citation: Bulat V, Šitum M, Maričić G, Škudar VL, Kovačević M, et al. (2014) Idiopathic Guttate Hypomelanosis: A Comprehensive Overview. Pigmentary Disorders 1:150. doi: 10.4172/2376-0427.1000150

Page 4 of 4

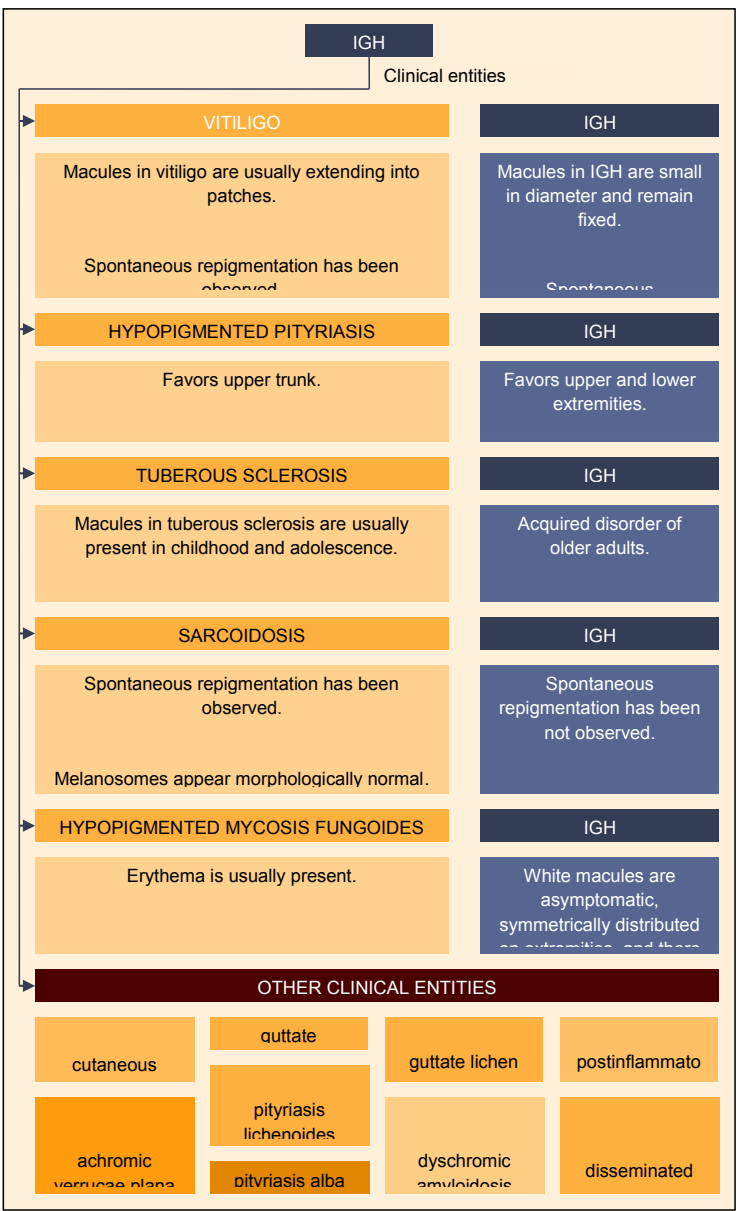

Figure 8: Differential diagnosis of IGH.

\section{References}

1. Ortonne JP, Perrot H (1980) Idiopathic guttate hypomelanosis. Arch Dermatol116: 664-668.

2. Savall R, Ferrandiz C, Ferrer I, Peyri J (1980) Idiopathic guttate hypomelanosis. Br J Dermatol 103: 635-642.

3. Flabella R (1988) Idiopathic guttate hypomelanosis. Dermatol Clin 6: 241-247.

4. Ortonne JP, Passeron T (2014) Vitiligo and other disorders of hypopigmentation. In: Dermatology. Elsevier, New Haven, Mosby 1023-1048.

5. Kim SK, Kim EH, Kang HY, Lee ES, Sohn S, et al. (2010) Comprehensive understanding of idiopathic guttate hypomelanosis: clinical and histopathological correlation. Int J Dermatol. 49: 162-166.

6. Flabella R, Escobar C, Giraldo N, Rovetto P, Gil J, et al. (1987) On the pathogenesis of idiopathic guttate hypomelanosis. J Am Acad Dermatol 16: $35-44$

7. Ploysangam T, Dee-Ananlap S, Suvanprakorn P (1990) Treatment of idiopathic guttate hypomelanosis with liquid nitrogen: light and electron microscopic studies. J Am Acad Dermatol 3: 681-684.

8. Hara M, Yaar M, Byers HR, Goukassian D, Fine RE, et al. (2000) Kinesin participates in melanosomal movement along melanocyte dendrites. J Invest Dermatol 114: 438-443.

9. Tsao AS, Kantarijan H, Cortes J, O`Brien S, Talpaz M (2003) Imatinib mesylate causes hypopigmentation in the skin. Cancer 98: 2483-2487.

10. Bolognia JL, Shapiro PE (1995) Albinism and other disorders of hypopigmentation.In: Cutaneous Medicine and Surgery. W.B. Saunders, Philadelphia 1219-1232.
11. Bolognia JL (1999) Clinical approach to leukoderma. Int J Dermatol 38: 568572

12. Relyveld GN, Menke HE, Westerhof W (2007) Progressive macular hypomelanosis: an overview. Am J Clin Dermatol 8: 13-19.

13. Broding HC, Monsé C, Brüning T, Fartasch M (2011) Induction of occupational leukoderma and vitiligo. Can butylated hydroxytoluene induce vitiligo similary to p-tert-butylphenol? Hautarzt $62: 209-214$

14. Solano F, Briganti S, Picardo M, Ghanem G (2006) Hypopigmenting agents: an updated review on biological, chemical and clinical aspects. Pigment Cell Res 19: 550-571.

15. Jimbow K (1997) Tuberous sclerosis and guttate leukodermas. Semin Cutan Med Surg 16: 30-35.

16. Jadotte YT, Janniger CK (2011) Pityriasis alba revisited: perspectives on an enigmatic disorder of childhood 87: 66-72.

17. Ruiz Maldonado R Orozco-Covarrubias M (1997) Postinflammatory hypopigmentation and hyperpigme-ntation. Semin Cutan Med Surg 16: 36-43.

18. Vachiramon V, Thadanipon K (2011) Postinflammatory hypopigmentation. Clin Exp Dermatol 36: 708-714 